\title{
Outage Performance of Two-Hop OFDM with Index Modulation and Multi-Carrier Relay Selections
}

\author{
Shuping Dang, Student Member, IEEE, Gaojie Chen, Member, IEEE and Justin P. Coon, Senior Member, IEEE
}

\begin{abstract}
In this paper, we propose a two-hop orthogonal frequency-division multiplexing with index modulation (OFDMIM) system, which is assisted by multiple relays and employs multi-carrier relay selection to enhance the system reliability. In particular, two commonly used multi-carrier relay selection schemes are considered, i.e., bulk and per-subcarrier relay selections, and the decode-and-forward (DF) relaying is adopted as the forwarding protocol for the proposed system. The analysis of average outage probability of the proposed system is given in closed form and substantiated by numerical results generated by Monte Carlo simulations. By the analytical and numerical results provided, we can observe that the full diversity (equal to the number of relays) can be achieved by applying both bulk and per-subcarrier relay selections in two-hop OFDM-IM systems.
\end{abstract}

Index Terms-Index modulation, orthogonal frequencydivision multiplexing (OFDM), multi-carrier relay selection, twohop relay system, decode-and-forward (DF) relaying.

\section{INTRODUCTION}

$\mathbf{C}$ ONVENTIONALLY, amplitude and phase modulation (APM) scheme, e.g., $M$-ary phase-shift keying (PSK) and $M$-ary quadrature amplitude modulation (QAM), is applied in communication systems to map a sequence of information bits to a constellation symbol, which has a unique amplitude and phase. Such a constellation symbol can be represented by a point in the complex plane and has a bijective mapping relation with the corresponding bit sequence [1]. However, with a sharp increase in required reliability and data transmission rate, these conventional amplitude and phase modulation (APM) schemes are less able to fulfill the service requirements for next generation networks, and thereby new modulation schemes with different principles have been investigated in recent years [2], [3]. A new modulation approach termed OFDM with index modulation (OFDM-IM) was proposed to add one extra modulation dimension in addition to the classic amplitude and phase dimensions in APM schemes [4]. Primary research has confirmed the superior performance of OFDM-IM in terms of transmission rate and fidelity in several important scenarios [5], [6]. Meanwhile, cooperative transmission has been proved to be able to enhance the reliability and extend the network coverage of next generation networks. To fill the gap between OFDM-IM and cooperative transmission, we proposed and analyzed an adaptive OFDM-IM scheme for two-hop relay networks in [7], in which a two-hop OFDMbased system assisted by a single decode-and-forward (DF)

This work was supported by the SEN grant (EPSRC grant number EP/N002350/1) and the grant from China Scholarship Council (No. 201508060323)

S. Dang and J.P. Coon are with the Department of Engineering Science, University of Oxford, Oxford, U.K., OX1 3PJ (e-mail: \{shuping.dang, justin.coon\}@eng.ox.ac.uk).

G. Chen is with the Department of Engineering, University of Leicester, Leicester, U.K., LE1 7RH (email: gaojie.chen@leicester.ac.uk). relay is considered. In addition, we also formulate the power allocation problem for the adaptive OFDM-IM scheme in twohop relay networks and obtain a suboptimal solution based on the Karush-Kuhn-Tucker (KKT) conditions [8].

This contribution significantly extends the work in [7], [8], which focused on the case where there exists only one relay. Note that such a restricted approach cannot fully exploit the performance enhancement offered by cooperative transmission. Hence, we propose a two-hop OFDM-IM system assisted by multiple relays and performing multi-carrier relay selections in this paper. In particular, the DF relaying protocol is adopted at all relays and two commonly used multicarrier relay selection schemes, i.e. bulk and per-subcarrier relay selections are considered in this paper. Based on these considerations, we propose a new diversity technique for twohop OFDM-IM in the spatial domain, which is provided by multi-carrier relay selection. We also analyze the average outage probability of the proposed two-hop OFDM-IM system performing bulk and per-subcarrier relay selections and prove that the full diversity equal to the number of relays can be achieved in the proposed system. All analytical results are given in closed form.

\section{SySTEM MODEL}

\section{A. System Framework of Two-Hop OFDM-IM}

In this paper, we consider a two-hop cooperative OFDMIM system with a single pair of source and destination and $N_{S}$ subcarriers, which is assisted by $N_{R}$ DF relays. The sets of subcarriers and relays are denoted by $\mathcal{N}_{S}$ and $\mathcal{N}_{R}$, respectively. In order to address the zero-active subcarrier dilemma in subcarrier activation mapping ${ }^{1}$, we stipulate that there exists a special subcarrier termed the control subcarrier, which will always be active for APM symbol transmission purposes, so that some crucial information for system coordinations, e.g., synchronization, can always be transmitted. We specify that $\mathbf{b}_{C}$ is the bit sequence required to be modulated by an APM symbol conveyed on the control subcarrier; $\mathbf{b}_{S}(k)$ is the bit sequence required to be modulated by the subcarrier activation pattern, where $k \in \mathcal{K}=\left\{1,2,3, \ldots, 2^{N_{S}-1}\right\}$ and $\mathcal{K}$ is the set of all subcarrier activation patterns; $\mathbf{b}_{M}\left(m_{t}\right)$ is the bit sequence required to be modulated by an APM symbol conveyed on the $t$ th active subcarrier; $m_{t} \in \mathcal{M}=\{1,2, \ldots, M\}$, where $\mathcal{M}$ denotes the set of APM constellation symbols and $M$ is the APM order; Therefore, in each instant, a variable-length equiprobable stream $\left[\mathbf{b}_{C}, \mathbf{b}_{S}(k), \mathbf{b}_{M}\left(m_{1}\right), \mathbf{b}_{M}\left(m_{2}\right), \ldots, \mathbf{b}_{M}\left(m_{N_{A}(k)}\right)\right]$ is intended to be transmitted at the source, where $0 \leq$

\footnotetext{
${ }^{1}$ This dilemma is defined as all subcarriers are required to be inactive and emerges in OFDM-IM systems with a variable number of active subcarriers. More details can be found in [7].
} 


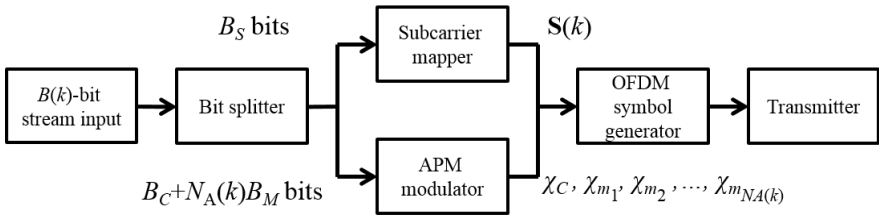

Fig. 1: System block diagram of the mapping process.

$N_{A}(k) \leq N_{S}-1$ is the number of active subcarriers except for the control subcarrier, when the $k$ th activation pattern is chosen. Supposing that the lengths of $\mathbf{b}_{C}, \mathbf{b}_{S}(k)$ and $\mathbf{b}_{M}\left(m_{t}\right)$ are $B_{C}, B_{S}$ and $B_{M}$, the length of the entire variable-length stream is $B(k)=B_{C}+B_{S}+N_{A}(k) B_{M}$.

Meanwhile, it is assumed that a single bit in $\mathbf{b}_{S}(k)$ represents the activation state of a given subcarrier in an on-off keying (OOK) manner, and for a given $B_{S}$-bit stream $\mathbf{b}_{S}(k)$, the subcarriers having the same sequence numbers with bits ' 1 ' will be activated, on which $N_{A}(k)$ different APM symbols are transmitted for multiplexing purposes. By this setting, we have $B_{S}=N_{S}-1$ and $N_{A}(k)$ is equal to the hamming weight of $\mathbf{b}_{S}(k)$. Mathematically, the activation state matrix of the proposed OFDM-IM system can be designed as

$$
\mathbf{S}(k)=\operatorname{diag}\left\{1, b_{S}(k, 1), b_{S}(k, 2), \ldots, b_{S}\left(k, N_{S}-1\right)\right\},
$$

where $b_{S}\left(k, n_{S}\right)$ is either ' 0 ' or ' 1 ' denoting the $n_{S}$ th entry in $\mathbf{b}_{S}(k), \forall n_{S} \in \mathcal{T}=\left\{1,2, \ldots, N_{S}-1\right\}$. The first element in $\mathbf{S}(k)$ is ' 1 ', which refers to the control subcarrier. Consequently, the entire $B(k)$-bit stream is now modulated to a $M$-ary constellation symbol $\chi_{C}$ conveyed on the control subcarrier, $N_{A}(k) M$-ary constellation symbols $\left\{\chi_{m_{t}}\right\}$ conveyed on other $N_{A}(k)$ active subcarriers and the activation state matrix $\mathbf{S}(k)$. We depict the system block diagram of the mapping process in Fig. 1.

In this paper, it is assumed that there is no direct transmission link between source and destination due to deep fading or severe propagation attenuation and a half-duplex DF forwarding protocol is adopted at all relays. Therefore, two orthogonal temporal phases are necessary for completing the transmission from source to destination. In addition, perfect synchronization in time and frequency domains and perfect channel estimation are supposed, such that full CSI is accessible at all nodes.

\section{B. Channel Model}

All wireless channels in the same hop are assumed to be independent and identically distributed (i.i.d.) Rayleigh fading channels in frequency and space, and their channel gains are thus exponentially distributed with average channel gain $\mu_{i}$, where $i \in\{1,2\}$ denotes the $i$ th hop. As a result, $\forall n_{R} \in \mathcal{N}_{R}$ and $n_{S} \in \mathcal{N}_{S}$, the probability density function (PDF) and the cumulative distribution function (CDF) of the channel gain $\left|h_{i}\left(n_{R}, n_{S}\right)\right|^{2}$ can be written as [1]

$$
f_{h_{i}}(s)=\exp \left(-s / \mu_{i}\right) / \mu_{i} \Leftrightarrow F_{h_{i}}(s)=1-\exp \left(-s / \mu_{i}\right) .
$$

Also, to facilitate the analysis of two-hop networks, we need to obtain the PDF and CDF of a link gain $\left|l\left(n_{R}, n_{S}\right)\right|^{2}=$ $\min \left\{\left|h_{1}\left(n_{R}, n_{S}\right)\right|^{2},\left|h_{2}\left(n_{R}, n_{S}\right)\right|^{2}\right\}$ of the $n_{S}$ th subcarrier forwarded by the $n_{R}$ th relay. By (2) and fundamentals of probability theory, we can easily have the CDF of the link gain by $F_{l}(s)=1-\left(1-F_{h_{1}}(s)\right)\left(1-F_{h_{2}}(s)\right)$.

\section{APM Scheme}

Because it has been proved that for systems employing IM [9], a constant-envelope APM can provide a higher power efficiency and reduce the complexity for symbol decoding, we employ $M$-PSK as the APM scheme in this paper. As a result of $M$-PSK, we can normalize the APM symbol conveyed on active subcarriers by $\chi_{C} \chi_{C}^{*}=1$ and $\chi_{m_{t}} \chi_{m_{t}}^{*}=1, \forall m_{t} \in \mathcal{M}$.

\section{Signal Transmission and Reception}

In this subsection, let us concentrate on the signal transmission and reception. To transmit $\mathbf{b}(k)$, we first need to construct the transmit OFDM block by a $N_{S}$-point inverse fast Fourier transform (IFFT) and insert a cyclic prefix (CP) with sufficient length in the block as a guard interval to prevent inter-symbol interference (ISI). By such a processing, the OFDM signaling model can be considered in a per-subcarrier manner, and the $N_{S} \times 1$ transmit OFDM block in frequency domain without interblock interference can be expressed as

$$
\mathbf{x}(k)=\left[\chi_{C}, x(1), \ldots, x\left(N_{S}-1\right)\right]^{T} \in \mathbb{C}^{N_{S} \times 1},
$$

where $(\cdot)^{T}$ denotes the matrix transpose operation;

$$
x\left(n_{S}\right)= \begin{cases}\chi_{m_{n_{S}}}, & n_{S} \in \mathcal{N}_{A}(k) \\ 0, & \text { otherwise }\end{cases}
$$

corresponds to the constellation symbol transmitted on the $n_{S}$ th subcarrier, and $\mathcal{N}_{A}(k) \subseteq \mathcal{T}$ is the subset of $N_{A}(k)$ active subcarriers out of $N_{S}-1$ remaining subcarriers except for the control subcarrier when $\mathbf{b}_{S}(k)$ is transmitted. Therefore, the received signal in the $i$ th hop can be written as [5]

$$
\begin{aligned}
& \mathbf{y}_{i}(k, \mathcal{L})=\sqrt{\frac{P_{t}}{1+N_{A}(k)}} \mathbf{H}_{i}(\mathcal{L}) \mathbf{x}(k)+\mathbf{w}_{i} \in \mathbb{C}^{N_{S} \times 1} \\
& =\left[y_{i}\left(n_{R}(C), C\right), y_{i}\left(n_{R}(1), 1\right), \ldots, y_{i}\left(n_{R}\left(N_{S}-1\right), N_{S}-1\right)\right]^{T},
\end{aligned}
$$

where $P_{t}$ is a uniform transmit power adopted at both source and relays; $\mathbf{w}_{i}=\left[w_{i}(C), w_{i}(1), \ldots, w_{i}\left(N_{S}-1\right)\right]^{T} \in \mathbb{C}^{N_{S} \times 1}$ denotes the vector of independent complex additive white Gaussian noise (AWGN) samples on each subcarrier at the $i$ th hop, whose entries are independently distributed according to $\mathcal{C N}\left(0, N_{0}\right)$, and $N_{0}$ is the noise power; $\mathbf{H}_{i}(\mathcal{L})=$ $\operatorname{diag}\left\{h_{i}\left(n_{R}(C), C\right), h_{i}\left(n_{R}(1), 1\right), \ldots, h_{i}\left(n_{R}\left(N_{S}-1\right), N_{S}-\right.\right.$ 1) $\} \in \mathbb{C}^{N_{S} \times N_{S}}$ is a $N_{S} \times N_{S}$ diagonal channel state matrix for the $i$ th hop; $\mathcal{L}=\bigcup_{n_{S} \in \mathcal{N}_{S}}\left\{n_{R}\left(n_{S}\right)\right\}$ denotes the set of selected relays and $n_{R}\left(n_{S}\right)$ represents the index of the relay forwarding the $n_{S}$ th active subcarrier, $\forall n_{S} \in \mathcal{N}_{S}$.

Because of the normalization of APM symbols, the received signal-to-noise ratio (SNR) of the entries in the received signal vector $\mathbf{y}_{i}(k)$ can be expressed as $\gamma_{i}\left(n_{R}(C), C\right)=\frac{P_{t}\left|h_{i}\left(n_{R}(C), C\right)\right|^{2}}{\left(1+N_{A}(k)\right) N_{0}}$ for the received signal transmitted on the control subcarrier and $\gamma_{i}\left(n_{R}\left(n_{S}\right), n_{S}\right)=$ $\frac{P_{t} b_{S}\left(k, n_{S}\right)\left|h_{i}\left(n_{R}\left(n_{S}\right), n_{S}\right)\right|^{2}}{\left(1+N_{A}(k)\right) N_{0}}, \forall n_{s} \in \mathcal{T}$ for other active subcarriers. Also, considering the overall performance in two-hop 
networks and the application of DF relaying, the end-to-end SNR for a certain subcarrier can be expressed

$$
\gamma\left(n_{R}\left(n_{S}\right), n_{S}\right)=\min \left\{\gamma_{1}\left(n_{R}\left(n_{S}\right), n_{S}\right), \gamma_{2}\left(n_{R}\left(n_{S}\right), n_{S}\right)\right\} .
$$

\section{Multi-Carrier Relay Selections and AVERage OUTAGE PROBABILITy}

\section{A. Multi-Carrier Relay Selection Schemes}

1) Bulk relay selection: The simplest multi-carrier relay selection scheme is called bulk relay selection, and this selection scheme selects only one relay to forward signals transmitted on all subcarriers. We can apply bulk relay selection to the proposed two-hop OFDM-IM system and the selected relay obtained through bulk relay selection can be expressed by

$$
\mathcal{L}_{\text {bulk }}=\underset{n_{R}\left(n_{S}\right) \in \mathcal{N}_{R}}{\arg \max }\left\{\min _{n_{S} \in \mathcal{N}_{\mathcal{S}}}\left|l\left(n_{R}\left(n_{S}\right), n_{S}\right)\right|^{2}\right\} .
$$

2) Per-subcarrier relay selection: In contrast, another basic multi-carrier relay selection scheme is called per-subcarrier relay selection, and it selects multiple relays in a per-subcarrier manner. Obviously, the per-subcarrier relay selection outperforms bulk relay selection in terms of outage performance, but its signaling overhead and system complexity are much higher ${ }^{2}$. We can determine the sets of selected relays obtained through the per-subcarrier relay selection scheme as

$$
\mathcal{L}_{p s}=\bigcup_{n_{S} \in \mathcal{N}_{\mathcal{S}}}\left\{\underset{n_{R}\left(n_{S}\right) \in \mathcal{N}_{R}}{\arg \max }\left|l\left(n_{R}\left(n_{S}\right), n_{S}\right)\right|^{2}\right\} .
$$

Note that, now relays are only able to decode and forward a portion of the information transmitted at the source, and the destination will retrieve the full information by properly decoding forwarded signals from all relays.

\section{B. Average outage probability}

To consider the overall performance of two-hop multicarrier systems as a whole, we can define the system-level outage event as follows.

Definition 1: An outage occurs when the SNR of any of the active subcarriers in either the first or the second hop falls below a preset outage threshold $s$.

As a result, the outage probability after performing multicarrier relay selections can be defined as

$$
P_{o}(s \mid k)=\mathbb{P}\left\{\bigcup_{i=1,2}\left\{\bigcup_{n_{S} \in \mathcal{A}(k)}\left\{\gamma\left(n_{R}\left(n_{S}\right), n_{S}\right)<s\right\}\right\}\right\},
$$

where $\mathbb{P}\{\cdot\}$ denotes the probability of the event enclosed and $\mathcal{A}(k)=\mathcal{N}_{A}(k) \cup\{C\}$. Finally, we can obtain the average outage probability over all subcarrier activation patterns by

$$
\bar{P}_{o}(s)=\underset{k \in \mathcal{K}}{\mathbb{E}}\left\{P_{o}(s \mid k)\right\},
$$

where $\mathbb{E}\{\cdot\}$ denotes the expected value of the random variable enclosed. The average outage probability is a key performance evaluation metric for system reliability.

\footnotetext{
${ }^{2}$ The system complexity referred to here is associated with the number of selected relays, because complexity is closely related to the selection and synchronization processes and would vary the system-level efficiency with different signaling overheads [10].
}

\section{Outage Performance Analysis}

\section{A. Bulk Relay Selection}

As the outage probability of multi-carrier systems is highly related to the number of active subcarriers, now we temporarily assume the $k$ th subcarrier activation pattern is chosen and there is $N_{A}(k)$ active subcarriers. Accordingly, we can derive the $a$ priori outage probability of the $n_{S}$ th subcarrier without conditioning on any selection by $\Phi(s \mid k)=F_{l}\left(\frac{s N_{0}\left(1+N_{A}(k)\right)}{P_{t}}\right)$. By (7) and order statistics, we can determine the a posteriori outage probability conditioning on bulk relay selection by

$$
P_{o}(s \mid k)=\left[1-(1-\Phi(s \mid k))^{1+N_{A}(k)}\right]^{N_{R}} .
$$

In addition, because $0 \leq N_{A}(k) \leq N_{S}-1$ and $\mathbf{b}_{S}(k)$ is equiprobable, the probability mass function (PMF) of $N_{A}(k)$ can be derived by $\phi_{N_{A}(k)}(\epsilon)=\left(\begin{array}{c}N_{S}-1 \\ \epsilon\end{array}\right) / 2^{N_{S}-1}$, where $(\cdot)$ denotes binomial coefficient. As a result of (10), we can thereby determine the average outage probability when the bulk relay selection is applied by

$$
P_{o}(s)=\sum_{N_{A}(k)=0}^{N_{S}-1} P_{o}(s \mid k) \phi_{N_{A}(k)}\left(N_{A}(k)\right) .
$$

To provide insight into the proposed system as well as the intrinsic relation among the outage performance and system parameters, we perform power series expansion at $P_{t} \rightarrow \infty$ and obtain the asymptotic expression of outage probability when the $k$ th subcarrier activation pattern is used as

$$
P_{o}(s \mid k) \sim\left[\frac{s N_{0}\left(1+N_{A}(k)\right)^{2}}{P_{t}}\left(\frac{1}{\mu_{1}}+\frac{1}{\mu_{2}}\right)\right]^{N_{R}} .
$$

Finally, we can also obtain the asymptotic expression of average outage probability by

$$
\begin{aligned}
P_{o}(s) \sim & {\left[\frac{s N_{0}}{P_{t}}\left(\frac{1}{\mu_{1}}+\frac{1}{\mu_{2}}\right)\right]^{N_{R}} } \\
& \times \sum_{N_{A}(k)=0}^{N_{S}-1} \phi_{N_{A}(k)}\left(N_{A}(k)\right)\left(1+N_{A}(k)\right)^{2 N_{R}},
\end{aligned}
$$

by which we can find the diversity to be $N_{R}$.

\section{B. Per-Subcarrier Relay Selection}

In a similar approach for studying the bulk relay selection, we can obtain the a posteriori outage probability conditioning on per-subcarrier relay selection by (8) and order statistics as

$$
P_{o}(s \mid k)=1-\left[1-(\Phi(s \mid k))^{N_{R}}\right]^{1+N_{A}(k)} .
$$

Consequently, we can employ (12) to obtain the average outage probability over all subcarrier activation patterns. Similarly, we also perform the power series expansion at $P_{t} \rightarrow \infty$ and the asymptotic expression of $P_{o}(s \mid k)$ is given by

$$
P_{o}(s \mid k) \sim\left(1+N_{A}(k)\right)\left[\frac{s N_{0}\left(1+N_{A}(k)\right)}{P_{t}}\left(\frac{1}{\mu_{1}}+\frac{1}{\mu_{2}}\right)\right]^{N_{R}} .
$$


Finally, we obtain the asymptotic expression of average outage probability when per-subcarrier relay selection is applied by

$$
\begin{aligned}
P_{o}(s) \sim & {\left[\frac{s N_{0}}{P_{t}}\left(\frac{1}{\mu_{1}}+\frac{1}{\mu_{2}}\right)\right]^{N_{R}} } \\
& \times \sum_{N_{A}(k)=0}^{N_{S}-1} \phi_{N_{A}(k)}\left(N_{A}(k)\right)\left(1+N_{A}(k)\right)^{N_{R}+1},
\end{aligned}
$$

by which we can also find the diversity to be $N_{R}$. By (14) and (17), it is thereby proved that the full diversity $N_{R}$ can be achieved by both relay selection schemes.

\section{NuMERICAL RESULTS}

In this section, we employ Monte Carlo simulations to substantiate the analysis presented in Section IV. To do so, we first normalize the system parameters as follows: $\mu_{1}=\mu_{2}=1$, $s=1$ and $N_{0}=1$. Then, we can vary the numbers of relays and subcarriers $N_{R}$ and $N_{S}$ to explore the relation between $P_{t} / N_{0}$ and the outage probability. We plot the relation between the ratio of transmit power to noise power $P_{t} / N_{0}$ and the outage probabilities defined in (10) for two-hop OFDMIM systems applying bulk and per-subcarrier relay selections. The simulation results are presented in Fig. 2 and Fig. 3 for both selection schemes, respectively. The analytical curves are plotted according to (11), (12) and (15). The asymptotic results are generated by (14) and (17). Meanwhile, we also take the two-hop OFDM-IM system that does not use relay selection, the conventional two-hop OFDM system with multicarrier relay selection and the cases with the variable-gain amplify-and-forward (VG-AF) relaying protocol as comparison benchmarks. From Fig. 2 and Fig. 3, we first verify the effectiveness of the analyses given in Section IV for outage performance. Also, it can be observed that the full diversity can be obtained by both multi-carrier relay selection schemes. Hence, the most efficient approach to enhance the reliability of two-hop OFDM-IM systems would be to increase the number of relays $N_{R}$. Besides, the proposed system using either bulk or per-subcarrier relay selection outperforms the comparison benchmarks that do not use relay selection, which confirms that multi-carrier relay selection can enhance the system reliability. Also, a better reliability can be achieved by the OFDM-IM scheme than that of the conventional OFDM scheme. Furthermore, DF relaying is better than VGAF relaying for two-hop OFDM-IM systems in terms of outage performance, which aligns with the results presented for conventional OFDM systems in [10].

\section{CONCLUSION}

In this paper, we proposed new diversity technique in space domain for two-hop OFDM-IM systems provided by multicarrier relay selections. Two fundamental and commonly used multi-carrier relay selection schemes and the DF relaying protocol are employed in this paper for analysis. We derived the closed-form expressions of exact outage probability for all cases as well as their asymptotic expressions when the transmit power goes large. The asymptotic results reveal that the full diversity equal to the total number of relays can be achieved in the proposed system.

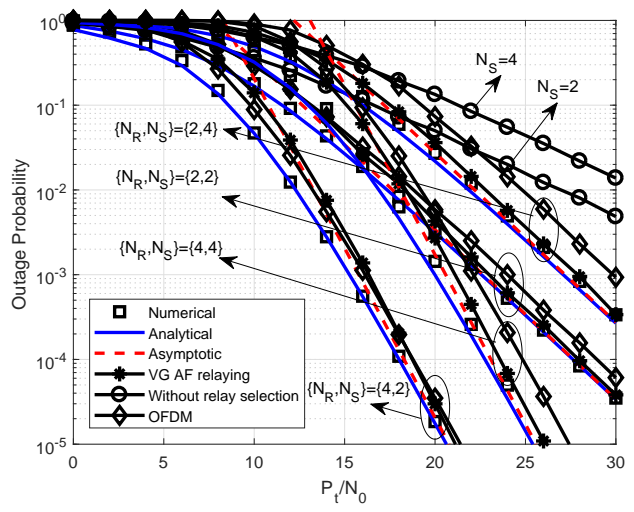

Fig. 2: Outage probability vs. $P_{t} / N_{0}(\mathrm{~dB})$ for bulk relay selection, given $N_{R} \in\{2,4\}$ and $N_{S} \in\{2,4\}$.

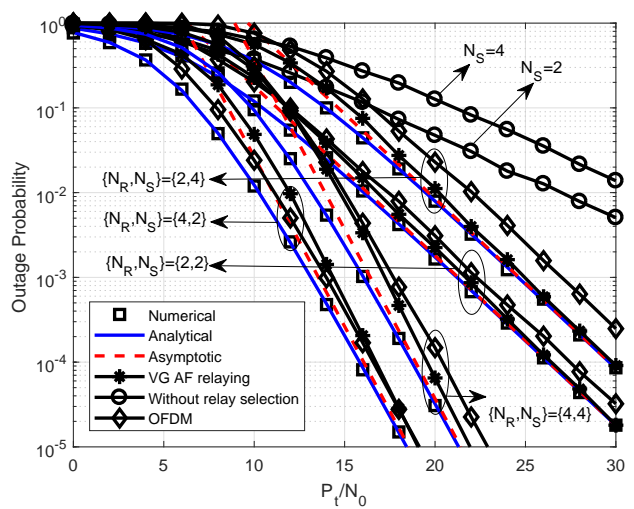

Fig. 3: Outage probability vs. $P_{t} / N_{0}(\mathrm{~dB})$ for per-subcarrier relay selection, given $N_{R} \in\{2,4\}$ and $N_{S} \in\{2,4\}$.

\section{REFERENCES}

[1] J. Proakis and M. Salehi, Digital Communications. McGraw-Hill Education, 2007.

[2] M. D. Renzo, H. Haas, A. Ghrayeb, S. Sugiura, and L. Hanzo, "Spatial modulation for generalized MIMO: challenges, opportunities, and implementation," Procs. of the IEEE, vol. 102, no. 1, pp. 56-103, Jan. 2014.

[3] E. Basar, M. Wen, R. Mesleh, M. D. Renzo, Y. Xiao, and H. Haas, "Index modulation techniques for next-generation wireless networks," IEEE Access, vol. 5, pp. 16693-16746, 2017.

[4] E. Basar, U. Aygolu, E. Panayrc, and H. V. Poor, "Orthogonal frequency division multiplexing with index modulation," IEEE Trans. on Signal Processing, vol. 61, no. 22, pp. 5536-5549, Nov. 2013.

[5] N. Ishikawa, S. Sugiura, and L. Hanzo, "Subcarrier-index modulation aided OFDM - will it work?" IEEE Access, vol. 4, pp. 2580-2593, May 2016.

[6] E. Basar, "Index modulation techniques for $5 \mathrm{G}$ wireless networks," IEEE Communications Magazine, vol. 54, no. 7, pp. 168-175, Jul. 2016.

[7] S. Dang, J. P. Coon, and G. Chen, "Adaptive OFDM index modulation for two-hop relay-assisted networks," IEEE Trans. on Wireless Communications, 2017.

[8] S. Dang, G. Chen, and J. P. Coon, "Power allocation for adaptive OFDM index modulation in cooperative networks," Singapore, 2017.

[9] M. Wen, X. Cheng, M. Ma, B. Jiao, and H. V. Poor, "On the achievable rate of OFDM with index modulation," IEEE Trans. on Signal Processing, vol. 64, no. 8, pp. 1919-1932, Apr. 2016.

[10] S. Dang, J. P. Coon, and G. Chen, "An equivalence principle for OFDMbased combined bulk/per-subcarrier relay selection over equally spatially correlated channels," IEEE Trans. on Veh. Tech., vol. 66, no. 1, pp. 122133, Jan. 2017. 